\title{
Evaluation of methicillin resistance in Staphylococcus intermedius isolated from dogs
}

\author{
V. Meucci • M. Vanni • L. Guardabassi • A. Moodley • \\ G. Soldani • L. Intorre
}

Published online: 2 May 2010

(C) Springer Science+Business Media B.V. 2010

\begin{abstract}
Methicillin and multi-drug resistance were investigated in 136 Staphylococcus intermedius strains of canine origin. The large majority of isolates were found to be mecAnegative by polymerase chain reaction, whereas only four strains were positive for the $m e c A$ gene. All mecA-positive strains were confirmed as methicillin-resistant by complementary tests, except for oxacillin disk diffusion, which yielded one false-negative result. A significantly higher resistance to fusidic acid, lincosamides, and cotrimoxazole was observed in methicillin-resistant $S$. intermedius (MRSI) compared with methicillinsusceptible strains. Although the prevalence of MRSI in dogs appeared to be low, methicillin resistance was confirmed to be associated with multi-drug resistance, suggesting the importance of antimicrobial susceptibility testing of canine $S$. intermedius strains.
\end{abstract}

Keywords Antimicrobials $\cdot$ Dog $\cdot$ Methicillin resistance $\cdot$ Staphylococci

$\begin{array}{ll}\text { Abbreviations } \\ \text { MRSI } & \text { methicillin-resistant Staphylococcus intermedius } \\ \text { MSSI } & \text { methicillin-susceptible Staphylococcus intermedius } \\ \text { PBP2a } & \text { penicillin-binding protein 2a } \\ \text { PCR } & \text { Polymerase Chain Reaction }\end{array}$

\section{Introduction}

Staphylococcus intermedius is responsible for canine pyoderma, a widespread inflammatory skin disease (Jones et al. 2007). Although antimicrobial agents such as cephalosporins, aminopenicillins, and fluoroquinolones have proven their efficacy in the treatment of infections caused by $S$. intermedius, an increase in multidrug resistance has been observed

V. Meucci $(\bowtie) \cdot M$. Vanni $\cdot$ G. Soldani $\cdot$ L. Intorre

Dipartimento di Clinica Veterinaria, Università di Pisa, viale delle Piagge 2, 56124 Pisa, Italy

e-mail: vmeucci@vet.unipi.it

L. Guardabassi · A. Moodley

Department of Veterinary Disease Biology, Faculty of Life Science, University of Copenhagen, Stigbøjlen 4, 1870 Frederiksberg, C, Denmark 
in recent years, mainly due to the rapid spread of methicillin-resistant $S$. intermedius (MRSI) (Sasaki et al. 2005; Hauschild and Wójcik 2007). Methicillin resistance in staphylococci is linked to the presence of the mecA gene that encodes the penicillin-binding protein 2a (PBP2a) with a reduced affinity for penicillins. Polymerase chain reaction (PCR)-based molecular techniques for $m e c A$ detection are generally considered reference techniques (Van Duijkeren et al. 2004; Pedersen et al. 2007); additional methods include the oxacillin agar-screening test, the agar diffusion method with oxacillin or cefoxitin disks, and a latex agglutination test for PBP2a detection. The purpose of the present study was to evaluate the presence of methicillin resistance in S. intermedius strains isolated from dogs, comparing the results obtained using three phenotypic techniques with those of a molecular method, and to investigate the presence of multi-resistant strains.

\section{Materials and methods}

One hundred thirty-six $S$. intermedius strains isolated from dogs referred to the Department of Veterinary Clinics, University of Pisa, previously identified using a PCR-based method, were included in this study (Jousson et al. 2007). The presence of the mecA gene was detected by a PCR-based technique as described by Zhang et al. (2004), and results were confirmed by the following complementary tests: growth on oxacillin-containing media $(2 \mathrm{mg} / \mathrm{L})$, agar diffusion with oxacillin disks $(5 \mu \mathrm{g})$, and latex agglutination for PBP2a detection (MRSA Slidex Detection, bioMerieux, Marcy l'Etoile, France). Sensitivity against 19 antimicrobial agents (fusidic acid, amikacin, amoxicillin/clavulanic acid, cephalexin, cefoperazone, clindamycin, chloramphenicol, cotrimoxazole, enrofloxacin, erythromycin, fosfomycin, gentamicin, lincomycin, marbofloxacin, moxifloxacin, norfloxacin, penicillin G, pristinamycin, and rifampicin) was evaluated using the disk diffusion method on Muller Hinton agar plates (Oxoid, Milano, Italy). Strains were classified as susceptible or resistant to antimicrobials tested in accordance with breakpoints proposed by the Comité de l'Antibiogramme de la Société Française de Microbiologie (CASFM). The proportion of multi-resistance between MRSI and methicillin-susceptible $S$. intermedius (MSSI) was compared using the Fisher's exact test (differences were considered significant for $P<0.05$ ).

\section{Results}

A total of 132 out of $136 \mathrm{~S}$. intermedius strains (97.1\%) were mecA-negative, whereas only 4 out of 136 strains (2.9\%) were positive for the mecA gene (Table 1). All mecA-positive

Table 1 Methicillin resistance in 136 S. intermedius strains isolated from dogs (PCR vs. phenotypic methods)

Number of strains with indicated results

Oxacillin agar screening test Oxacillin disk diffusion test Latex agglutination test

\begin{tabular}{lllllll}
\hline mecA PCR & Growth & No growth & Resistant & Susceptible & Positive & Negative \\
positive $(n=4)$ & $4(100)^{\mathrm{a}}$ & 0 & $3(75)^{\mathrm{a}}$ & 1 & $4(100)^{\mathrm{a}}$ & 0 \\
negative $(n=132)$ & 0 & $132(100)^{\mathrm{b}}$ & 1 & $131(99.2)^{\mathrm{b}}$ & 1 & $131(99.2)^{\mathrm{b}}$ \\
\hline
\end{tabular}

\footnotetext{
${ }^{\text {a }}$ Sensitivity

${ }^{\mathrm{b}}$ Specificity
} 
Table 2 Susceptibility of 136 S. intermedius strains against 19 selected antimicrobials

\begin{tabular}{|c|c|c|c|}
\hline \multirow[t]{2}{*}{ Antimicrobial } & \multicolumn{2}{|c|}{ Susceptibility (\%) } & \multirow{2}{*}{$\begin{array}{l}\text { Statistical analysis }{ }^{\mathrm{a}} \\
P\end{array}$} \\
\hline & MRSI $(n=4)$ & $\operatorname{MSSI}(n=132)$ & \\
\hline Fusidic acid & 75 & 100 & 0.029 \\
\hline Amikacin & 100 & 100 & - \\
\hline Amoxicillin/Clavulanic acid & 0 & 100 & $<0.0001$ \\
\hline Cephalexin & 0 & 100 & $<0.0001$ \\
\hline Cefoperazone & 0 & 100 & $<0.0001$ \\
\hline Clindamycin & 50 & 93.2 & 0.032 \\
\hline Chloramphenicol & 75 & 87.9 & 0.41 \\
\hline Cotrimoxazole & 50 & 97 & 0.009 \\
\hline Enrofloxacin & 50 & 100 & 0.0007 \\
\hline Erythromycin & 50 & 83.3 & 0.14 \\
\hline Fosfomycin & 100 & 99.2 & 1 \\
\hline Gentamicin & 100 & 98.5 & 1 \\
\hline Lincomycin & 15 & 89.4 & 0.0061 \\
\hline Marbofloxacin & 50 & 100 & 0.0007 \\
\hline Moxifloxacin & 100 & 100 & - \\
\hline Norfloxacin & 50 & 100 & 0.0007 \\
\hline Penicillin G & 0 & 37.9 & 0.29 \\
\hline Pristinamycin & 100 & 100 & - \\
\hline Rifampicin & 100 & 99.2 & 1 \\
\hline
\end{tabular}

${ }^{\text {a }}$ Fisher's exact test (significant differences for $P<0.05$ )

Table 3 Multiple resistance in 25 S. intermedius strains

\begin{tabular}{llcc}
\hline Multi-resistance pattern & MRSI & MSSI & Total \\
\hline BLs + FQs + LAs + MLs + SAs & 2 & 0 & 2 \\
BLs + LAs + MLs + APs + RMs & 0 & 1 & 1 \\
BLs + LAs + MLs + SAs + AGs & 0 & 1 & 1 \\
BLs + LAs + APs + FD & 1 & 0 & 1 \\
BLs + LAs + MLs + AGs & 0 & 1 & 1 \\
BLs + LAs + MLs + APs & 0 & 6 & 6 \\
BLs + LAs + APs & 0 & 1 & 1 \\
BLs + LAs + MLs & 0 & 5 & 5 \\
BLs + MLs + APs & 0 & 1 & 6 \\
BLs + MLs + SAs & 0 & $22^{\mathrm{a}}$ & 1 \\
Total & $3^{\mathrm{a}}$ & & 25 \\
\hline
\end{tabular}

$\mathrm{BLs}=\beta$-lactams, FQs $=$ fluoroquinolones, Las $=$ lincosamides, MLs $=$ macrolides, Sas $=$ sulfonamides, Aps $=$ amphenicols, Ags $=$ aminoglycosides, $\mathrm{FD}=$ fusidic acid, $\mathrm{RMs}=$ rifamycins

${ }^{\text {a }} P=0.019$ 
strains were confirmed as MRSI by the oxacillin agar-screening test and the latex agglutination test, while the oxacillin disk diffusion test gave a false-negative result. Among the mecA-negative isolates, one strain resulted positive for PBP2a detection and a second strain was found resistant by the disk diffusion method. Susceptibility tests showed significantly lower rates of susceptibility in MRSI compared with MSSI to fusidic acid, amoxicillin/clavulanic acid, cephalexin, cefoperazone, clindamycin, cotrimoxazole, enrofloxacin, lincomycin, marbofloxacin, and norfloxacin (Table 2). Multidrug resistance (resistance to at least three different antimicrobial classes) was detected in 25 out of 136 S. intermedius strains (18.4\%), resulting in significantly more frequent MRSI $(75 \%)$ than MSSI (16.7\%) (Table 3).

\section{Discussion}

All phenotypic tests used in the present study showed high sensitivity and specificity compared with mecA-PCR; nevertheless, the obtained results indicated that the performance of different methods is preferable compared with a single test to discriminate between MRSI and MSSI. MRSI strains were more frequently associated with multiple resistance compared with MSSI, primarily for antimicrobials used to treat canine pyoderma, such as cephalosporins, amoxicillin/clavulanic acid, and fluoroquinolones, confirming the association between methicillin resistance and multiple resistance previously reported (Sasaki et al. 2005; Hauschild and Wójcik 2007). In conclusion, even though a very low prevalence of methicillin-resistant $S$. intermedius was observed, the results suggest the need for continuous monitoring of $S$. intermedius antimicrobial susceptibility, underlying the importance of prudent use of antimicrobials in companion animals.

\section{References}

Hauschild T, Wójcik A (2007) Species distribution and properties of staphylococci from canine dermatitis. Res Vet Sci 82:1-6

Jones RD, Kania SA, Rohrbach BW, Frank LA, Bemis DA (2007) Prevalence of oxacillin- and multidrugresistant staphylococci in clinical samples from dogs: 1,772 samples (2001-2005). J Am Vet Med Assoc 230:221-227

Jousson O, Di Bello D, Vanni M, Cardini G, Soldani G, Pretti C, Intorre L (2007) Genotypic versus phenotypic identification of staphylococcal species of canine origin with special reference to Staphylococcus schleiferi subsp. coagulans. Vet Microbiol 123:238-244

Pedersen K, Pedersen K, Jensen H, Finster K, Jensen VF, Heuer OE (2007). Occurrence of antimicrobial resistance in bacteria from diagnostic samples from dogs. J Antimicrob Chemother 60:775-781

Sasaki A, Shimizu A, Kawano J, Wakita Y, Hayashi T, Ootsuki S (2005) Characteristics of Staphylococcus intermedius isolates from diseased and healthy dogs. J Vet Med Sci 67:103-106

Van Duijkeren E, Box AT, Heck ME, Wannet WJ, Fluit AC (2004) Methicillin-resistant staphylococci isolated from animals. Vet Microbiol 103:91-97

Zhang K, Sparling J, Chow BL, Elsayed S, Hussain Z, Church DL, Gregson DB, Louie T, Conly JM (2004) New quadriplex PCR assay for detection of methicillin and mupirocin resistance and simultaneous discrimination of Staphylococcus aureus from coagulase-negative staphylococci. J Clin Microbiol 42:4947-4955 